\title{
Die Stiftung als Dienstleister
}

\section{Das Wohlfahrtswerk für Baden-Württemberg versteht sich als Unternehmer und Innovator}

\author{
Ingrid Hastedt
}

The Wohlfahrtswerk für Baden-

Württemberg goes back to an initiative of Queen Katharina of Württemberg. In 1817, she founded welfare associations and inaugurated a central administration with a coordinating function. In 1972 the state released the organisation, transforming it into a foundation under private law. The set task was to provide high-quality services for people in need, based on sound economic standing and continuous innovation.

Le Wohlfahrtswerk für Baden-Württemberg remonte à une initiative de la reine Katharina du Wurtemberg. En 1817, elle a créé des associations de bienfaisance et a investi une administration centrale de la fonction de coordination. En 1972 l'Etat a déchargé l'organisation de ses fonctions, la transformant en une fondation de droit privé. La tâche assignée était de fournir des services de haute qualité aux personnes dans le besoin, basés sur une situation financière fiable et l'innovation constante.

Ingrid Hastedt ist Vorsitzende des Vorstandes des Wohlfahrtswerks für Baden-Württemberg. Die Stiftung ist ein großer sozialwirtschaftlicher Dienstleister und Herausgeber der Blätter der Wohlfahrtspflege. Internet http://www.wohlfahrtswerk.de
Das Woblfahrtswerk für Baden-Württemberg ist aus einer Initiative der Königin Katharina von Württemberg hervorgegangen, die im Jahre 1817 Wobltätigkeitsvereine gründete und eine koordinierende Zentralleitung einsetzte. Im Jahre 1972 wurde die Organisation aus der Verantwortung des Staates entlassen und in eine privatrechtliche Stiftung überfübrt - mit dem Auftrag, durch gutes Wirtschaften und kontinuierliche Innovation hochwertige Dienstleistungen für hilfesuchende Menschen anzubieten.

Die Stiftung ist eine gängige Rechtsform für Einrichtungsträger, die im Sozialbereich tätig sind. Bei Neugründungen von operativ tätigen Organisationen dominieren mittlerweile die gemeinnützige und die gewinnorientierte Gesellschaft mit beschränkter Haftung $(\mathrm{GmbH})$. Organisationen, die seit Jahrzehnten im Sozialbereich in der Rechtsform des eingetragenen Vereins tätig sind, gründen zunehmend Tochtergesellschaften mit beschränkter Haftung. Stiftungsgründungen von institutioneller Seite zielen heutzutage auf das Einwerben von Finanzierungsbeiträgen für soziale Dienstleistungen, die durch andere Rechtsträger erbracht werden. Inwieweit die Stiftung als Basis für unternehmerisches Handeln im Sozialbereich heute geeignet erscheint, kann am Beispiel des Wohlfahrtswerks für BadenWürttemberg erläutert werden.

\section{Die Gründungsmotive}

Beim Wohlfahrtswerk für Baden-Württemberg handelt es sich um eine Stiftung bürgerlichen Rechts. Sie wurde im Jahre 1972 per Beschluss des Landtags von Baden-Württemberg gegründet. Mit der Errichtung der Stiftung wurde eine damals seit über 150 Jahren bestehende, inhaltlich wechselhafte Tätigkeit in eine auf Kontinuität ausgerichtete Rechtsform überführt.
Die Wurzeln im Jahr 1816 waren dem Stiftungswesen bereits sehr nahe: Königin Katharina von Württemberg hatte die Gründung von örtlichen »Wohltätigkeitsvereinen " angeregt, um das zuvor unkoordinierte Spendenwesen zu bündeln und die »Zentralleitung für die freiwilligen Wohltätigkeitsvereine in Württemberg « zwecks landesweiter Koordination zielgerichteter, effizienter Mittelverwendung gegründet.

Nach den Rechtsformen als öffentlichrechtliche Körperschaft ab 1902 und unter dem Namen »Landeswohlfahrtswerk « seit 1956 als Anstalt öffentlichen Rechts, bedeutete die Gründung einer Stiftung bürgerlichen Rechts eine echte Privatisierung. Immobilien und Betrieb von sieben Altenhilfeeinrichtungen größtenteils in den 1960er-Jahren errichtet - mit damals 985 Bewohnern gingen in das Vermögen der neu gegründeten Stiftung über, die übrigen Rechte und Verbindlichkeiten des Landeswohlfahrtswerks ebenso. Im Vorfeld der Stiftungsgründung stand die Auflösung der gesamten Organisation zur Diskussion, da sich seit dem Ausbau der 1952 gegründeten "Liga der freien Wohlfahrtspflege in Baden-Württemberg « gewisse Doppelstrukturen in Tätigkeit und Selbstverständnis der Liga und des Landeswohlfahrtswerks ergeben hatten. Vor diesem Hintergrund waren die mit einer Stiftung verbundene Dauerhaftigkeit und der Verzicht auf einen öffentlich-rechtlichen Status bewusst gewählt.

Die Motivation zur Stiftungsgründung hing mit der dauerhaften Sicherung des Vermögens zusammen: Das einst große Vermögen, das sich aus Zustiftungen im Laufe der Geschichte seit Mitte des 19. Jahrhunderts speiste, war durch die Inflation der 1920er-Jahre und die Währungsreform 1948 zusammengeschmolzen. Der seit 1966 amtierende Verwaltungsratsvorsitzende und Erste Vorsitzende der Landessparkasse Staatsrat a. D. Dr. Max Fetzer verwies darauf, dass aus Respekt 
vor dem Willen der Stifterinnen und Stifter das verblieben Vermögen nicht verwirtschaftet werden dürfe. Er sah das Landeswohlfahrtswerk auf lange Sicht auf großzügige Spenden und staatliche Zuschüsse angewiesen. Diese Gefahr ergab sich u. a. aus der umfassenden Bautätigkeit der vorangegangenen Jahre.

Die Wirtschaftlichkeit des Betriebs der eigenen Altenheime bedurfte erheblicher Anstrengungen. Vermögensverzehr drohte deshalb auch durch Unterdeckung der Betriebskosten. Betriebszuschüsse aus dem Staatshaushalt hätten - wegen sich wandelnder politischer Prioritäten - keine dauerhafte Finanzierungssicherheit bedeutet. Die Finanzierung der Bauinvestitionen und Unwirtschaftlichkeit des Betriebs sind genauso aktuelle Herausforderungen für in öffentlicher Trägerschaft geführte Heime geblieben.

Heute ist ein Verkauf der Immobilien und die Abgabe des Betriebs an private Betreiber eine übliche Lösung solcher Probleme. (1) Für das Wohlfahrtswerk wurde im Jahre 1972 ein anderer Weg beschritten; dieser brachte mit sich, dass es keine Liquiditätsspritze für den Landeshaushalt durch den Verkauf von Immobilien gab. Es wurde auch kein fremder Betreiber für den Betrieb gesucht. Offensichtlich bestand Vertrauen in die Etablierung eines leistungsfähigen Managements, sofern die entsprechenden Rahmenbedingungen dafür geschaffen würden.

\section{Der Stiftungszweck}

Die Formulierung des Stiftungszwecks ist von zentraler Bedeutung für die tägliche Arbeit einer Stiftung. Die Änderung des Stiftungszwecks unterliegt der strengen Prüfung der Stiftungsaufsicht und muss dem Abgleich mit dem Stifterwillen auch Jahrhunderte später standhalten können.

Beim Wohlfahrtswerk für Baden-Württemberg ist der Stiftungszweck weiter gefasst, als es die operative Tätigkeit erforderlich macht. Eine weite Fassung des Stiftungszwecks begünstigt, dass sich das operative Wirken der Stiftung an die Erfordernisse der jeweiligen Zeit anpassen kann. Die im Jahre 1956 gefassten »Grundbestimmungen des Landeswohlfahrtswerks « beschränkten sich auf die damals aktuelle Tätigkeit und formulierten als Zweck u. a. »die Unterhaltung von Wohlfahrtseinrichtungen, insbesondere Wohnheimen für Arbeitende, Altersheimen, Alterspflegeheimen und Arbeitsstuben «. Die Stiftungsgründer haben bei der Formulierung des Stiftungszwecks nur bedingt die Möglichkeit eröffnet, inhaltliche Tätigkeitsschwerpunkte an die Erfordernisse der Zeit anpassen zu können: Der Passus fand 1972 leicht abgewandelt Eingang in die neue Stiftungssatzung: » die Unterhaltung von Wohlfahrtseinrichtungen, insbesondere von Heimen, Wohnheimen und Pflegeheimen für betagte Menschen sowie von Heimen und Werkstätten für Behinderte«. Im Jahre 1993 wurde dieser Auftrag mit »Unterhaltung und Betrieb von Wohlfahrtseinrichtungen " allgemeiner formuliert und 1996 wurde nach einer mittlerweile erfolgten Ausweitung der Standorte die »Gründung « solcher Einrichtungen im Satzungszweck ergänzt.

Das Beispiel zeigt: Die Formulierung eines Stiftungszwecks ist im Detail durchaus veränderbar. Allerdings ist der erklärte oder mutmaßliche Stifterwille immer zu berücksichtigen. Andere gängige Unternehmensformen im Sozialbereich bringen demgegenüber weniger inhaltliche Bindung mit sich: Ein eingetragener Verein kann mit einem Beschluss der Mitgliederversammlung den Vereinszweck ändern. Bei der daraufhin notwendigen Eintragung ins Vereinsregister folgt dann nur die gemeinnützigkeitsrechtliche Prüfung des neuen Vereinszwecks. Die Änderung des Gesellschaftszwecks einer $\mathrm{GmbH}$ lässt ebenfalls viel Freiheiten, sich inhaltlich neu auszurichten: Vor der Änderungseintragung des Gesellschaftszwecks in das Handelsregister wird nicht die neue inhaltliche Ausrichtung beurteilt, sondern lediglich, ob die Gesellschaft die branchenbezogenen Voraussetzungen erfüllt.

Die Tätigkeit der Stiftung Wohlfahrtswerk für Baden-Württemberg ist momentan nicht fördernd, sondern operativ mit einem Schwerpunkt in der Altenhilfe ausgerichtet. Inwieweit ist die Stiftung aus Sicht der Praxis auch für diese Konstellation eine geeignete Rechtsform?

Anlass für die Stiftungsgründung im Jahre 1972 war u. a. die Erwartung, dass zur künftigen Vermeidung des Vermögensverzehrs erhebliche Spenden und $\mathrm{Zu}$ schüsse erforderlich wären. Für das Spendensammeln zugunsten eigener Einrichtungen ist die Stiftung selbstredend geeignet. Vor der Stiftungsgründung im Jahre 1972 war das Wohlfahrtswerk für Baden-Württemberg aber auch operativ tätig, was den weiter drohenden Vermögensverzehr mit verursachte. Damals galt es nicht, etwas komplett Neues aufzubauen, sondern Zweckbetriebe in eine andere Rechtsform zu überführen.

\section{Die Unternehmensführung}

Wie geeignet ist die Rechtsform der Stiftung für Zwecke der wirtschaftlichen Betriebsführung von sozialen Diensten und Einrichtungen? Die unternehmerische Handlungsfreiheit ist zunächst eingeschränkt durch die Rahmenbedingun-

\section{Unternehmensstruktur}

\begin{tabular}{|c|c|c|c|}
\hline \multicolumn{4}{|c|}{$\begin{array}{c}\text { Wohlfahrtswerk für Baden-Württemberg } \\
\text { Stiftung bürgerlichen Rechts }\end{array}$} \\
\hline & & & \\
\hline $\begin{array}{c}\text { Silberburg } \\
\text { Hausdienste } \\
\text { GmbH } \\
(1995)\end{array}$ & $\begin{array}{l}\text { Wohlfahrts- } \\
\text { werk } \\
\text { Altenhilfe } \\
\text { gGmbH } \\
\text { (1998) }\end{array}$ & $\begin{array}{l}\text { Wohlfahrts- } \\
\text { werk Mana- } \\
\text { gement und } \\
\text { Service GmbH } \\
\quad(2006)\end{array}$ & $\begin{array}{l}\text { Wohlfahrts- } \\
\text { werk Bau- } \\
\text { und Immobi- } \\
\text { lienmanage- } \\
\text { ment GmbH } \\
\quad(2006)\end{array}$ \\
\hline $\begin{array}{l}\text { Reinigung, } \\
\text { Haustechnik }\end{array}$ & $\begin{array}{l}\text { Betrieb } \\
\text { Altenhilfe- } \\
\text { einrichtungen }\end{array}$ & $\begin{array}{l}\text { Verwaltungs-/ } \\
\text { Management-DL, } \\
\text { Personalentleihe }\end{array}$ & $\begin{array}{l}\text { Dienstleistung } \\
\text { rund um den } \\
\text { Bau }\end{array}$ \\
\hline
\end{tabular}

○) Wohlfahrtswerk für Baden-Württemberg, 2006 
gen der Gemeinnützigkeit. Damit zusammenhängende Aspekte sollen im Folgenden nicht angesprochen werden, denn sie gelten für Stiftungen wie für eingetragene Vereine oder gemeinnützige Gesellschaften gleichermaßen.

Die Maßstäbe guter Unternehmensführung finden sich im »Deutschen Corporate Governance Kodex « (2), der mit dem Ziel zukunftssichernder, nachhaltiger und wertorientierter Unternehmensführung von führenden Unternehmenslenkern zunächst für börsennotierte Unternehmen entwickelt wurde. Dabei werden u. a. die handelnden Organe und deren Beziehungen strukturell betrachtet. Als erster in Sozialbereich tätiger Dachverband hat das Diakonische Werk der EKD im Oktober 2005 eine Anpassung für diakonische Unternehmen verabschiedet. (3) Strukturen und Organisation von Leitung und Kontrolle sind bei den im Sozialbereich operativ tätigen Institutionen in der Tat sehr unterschiedlich geregelt.

Im Lichte dieses Diakonischen Corporate Governance Kodex (DGK) sind das Zusammenwirken von Vorstand und Aufsichtsgremium von Bedeutung. Der Diakonische Corporate Governance Kodex fordert, dass in der Satzung die Zuständigkeit für Geschäfte von grundlegender Bedeutung geregelt wird. Schon die Ursprungsversion der Stiftungsurkunde des Wohlfahrtswerks für Baden-Württemberg aus dem Jahre 1972 hat die Aufgaben des Aufsichtsgremiums abschlie- ßend aufgelistet und alle Geschäfte, die nicht dem Aufsichtsrat zugewiesen sind, als Verantwortung des Vorstands definiert. Dieser war in den ersten Stiftungsjahren jedoch eingeschränkter als heute. Die erhebliche Ausweitung der Tätigkeit des Wohlfahrtswerks für Baden-Württemberg in der Altenhilfe führte dazu, die Befugnisse des Vorstands mit der Zeit durch Satzungsänderungen auszudehnen. Die Tätigkeit des Aufsichtsgremiums wurde mit einer grundlegenden Satzungsreform im Jahre 1996 auf Überwachungs- und Beratungstätigkeit zugeschnitten. Hinzu kommen Entscheidungen über die Verfügung von Vermögenswerten, deren Werte so dimensioniert sind, dass die alltägliche Betriebsführung davon nicht berührt ist.

Mit der wachsenden Verantwortung des Vorstands des Wohlfahrtswerks für Baden-Württemberg wurde die Ausweitung der Zahl der Vorstandsmitglieder von vorher einer auf zwei Personen festgelegt. Dieser zweiköpfige Vorstand des Wohlfahrtswerks für Baden-Württemberg ist hauptamtlich tätig und führt die Geschäfte der Stiftung. Damit werden Probleme, die sich aus einem Informationsgefälle zwischen haupt- und ehrenamtlichen Vorstandsmitgliedern ergeben können, von vornherein vermieden. Im Alltag begünstigt die Konzentration der Aufsichtsratsfunktion auf Überwachung und Beratung sowie der Verzicht auf eine Splittung zwischen ehrenamtlichem Vor-

\section{Die Altenhilfeeinrichtungen des Wohlfahrtswerks}

\begin{tabular}{|l|l|} 
Else-Heydlauf-Stiftung, Stuttgart-Zuffenhausen \\
Altenburgheim, Stuttgart-Bad Cannstatt \\
Eduard-Mörike-Seniorenwohnanlage, Stuttgart-Süd \\
Ludwigstift, Stuttgart-West \\
Betreutes Wohnen Stuttgart-West \\
Generationenhaus West, Stuttgart-West \\
Haus am Weinberg, Stuttgart-Obertürkheim \\
Olgaheim, Stuttgart-West (Geschäftsbesorgung durch Wohlfahrtswerk) \\
\begin{tabular}{|l|l|} 
Haus am Kappelberg, Fellbach \\
Jakob-Sigle-Heim, Kornwestheim \\
Karl-Walser-Haus, Ludwigsburg \\
Haus am Fleinsbach, Filderstadt-Bernhausen \\
Seniorenwohnanlage In den Gärtlesäckern, Leinfelden-Echterdingen \\
Haus Heckengäu, Heimsheim
\end{tabular} \\
\hline
\end{tabular}

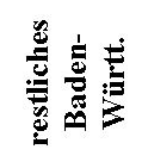

Kraichgauheim, Bad Schönborn

Lußhardtheim, Waghäusel-Kirrlach

Haus an der Steinlach, Mössingen

Haus im Park, Bisingen

() Wohlfahrtswerk für Baden-Württemberg, 2006

stand und hauptamtlicher Geschäftsführung (4) schnelle Entscheidungswege und klare Kompetenzverteilungen.

Die Zusammensetzung des Aufsichtsgremiums hat in der Stiftungszeit des Wohlfahrtswerks für Baden- Württemberg zahlreiche Veränderungen erfahren, die sich aus der jeweiligen Satzung ergaben. Ab Stiftungsgründung wurden einerseits Institutionen benannt, die ein Mitglied entsenden konnten. Daneben gab es natürliche Personen als geborene Mitglieder. Die in den damaligen "Verwaltungsrat « entsandten Mitglieder sind in der Regel mit Beendigung ihres Berufslebens aus dem Gremium ausgeschieden, was zu einem gewissen Ungleichgewicht führte, denn für die geborenen Mitglieder existierte keine Altersgrenze.

Mit der Satzungsreform im Jahr 1996 wurde der heutige Status von ausschließlich entsandten Mitgliedern erreicht. Bei der Benennung der Institutionen, die in den heutigen Aufsichtsrat entsenden, wurden Institutionen gewählt, die einerseits historisch bedingt mit dem Wohlfahrtswerk für Baden-Württemberg in Verbindung stehen. So ist das Land $\mathrm{Ba}-$ den-Württemberg ebenso berücksichtigt wie kommunale Gebietskörperschaften. Andererseits handelt es sich bei den Entsendern um Institutionen, die mit dem Sozialbereich zu tun haben. Im Wohlfahrtswerk für Baden-Württemberg wurde damit die Möglichkeit von Stiftungen genutzt, personenunabhängig Institutionen in die Verantwortung einzubinden und mitgestalten zu lassen.

Die Stiftung bewirkt auch, dass die Verwendung des Kapitals nicht der Willkür der Handelnden ausgesetzt ist, sondern dem Diktat des Stiftungszwecks. Trotzdem zeigt das Beispiel des Wohlfahrtswerks für Baden- Württemberg, dass flexible Mitgestaltungsmöglichkeiten für Institutionen existieren. Sie ergeben sich aus der Benennung von Personen für das Aufsichtsorgan, die wechseln können. Die Frage, welche Institution entsenden darf, ist aber dauerhaft verankert. Bei eingetragenem Verein und $\mathrm{GmbH}$ ist eine solche institutionelle Regelung der Mitgliedschaft im Aufsichtsorgan nicht möglich - es sei denn, es sind finanzielle Verflechtungen vorhanden wie im Fall der Gesellschafter einer $\mathrm{GmbH}$.

Es bleibt Stiftungen unbenommen, die Möglichkeiten anderer Rechtsformen auszunutzen: durch Gründung von Toch- 
tergesellschaften. Die Beteiligung an Gesellschaften ist möglich, soweit dafür einzubringende Gesellschaftsanteile nicht aus zweckgebundenen Mitteln der Stiftung entnommen werden. Die Stiftung Wohlfahrtswerk für Baden-Württemberg hat diese Möglichkeit genutzt für den Aufbau neuer Betriebe und neuer Dienstleistungszweige.

Derzeit gibt es vier hundertprozentige Tochtergesellschaften der Stiftung Wohlfahrtswerk für Baden- Württemberg, darunter eine gemeinnützige und drei gewinnorientierte. Hintergrund der Ausgründung der gemeinnützigen Tochtergesellschaft »Wohlfahrtswerk Altenhilfe $\mathrm{gGmbH}$ « war, dass die festgesetzten Pflegesätze für eine im Jahr 1997 neu eröffnete Altenpflegeeinrichtung in Anbetracht der Personalkosten und Arbeitsvertragsbedingungen bei der Stiftung keine Wirtschaftlichkeit des Betriebs erwarten ließen. So wurde eine Tochtergesellschaft eingegliedert, deren Arbeitsvertragsbedingungen an die Erfordernisse der Zeit angepasst gestaltet wurden. (5) Weitere, danach neu eröffnete Einrichtungen befinden sich ebenfalls in Trägerschaft dieser Tochtergesellschaft. Deren Zweck ist die umfassende Betreuung von hilfesuchenden, pflegebedürftigen und älteren Menschen, konzentriert sich somit auf eine Zielgruppe. Dieser Zweck überschneidet sich teilweise mit einem der Stiftungszwecke der Alleingesellschafterin, nämlich Unterhaltung und Betrieb von Wohlfahrtseinrichtungen.

Die Zwecke der anderen Tochtergesellschaften betreffen Dienstleistungen, die dem Betrieb der Stiftungseinrichtungen dienlich sind: hauswirtschaftliche Dienstleistungen, Verwaltungsservice, Personalvermittlung, Bau- und Immobilienmanagement. Auch diese Tochtergesellschaften verfügen über Tarifbedingungen, die der Wirtschaftlichkeit und Wettbewerbsfähigkeit des Gesamtunternehmens dienen. Mit den Tochtergesellschaften besteht daneben die Möglichkeit, sich operativ anders auszurichten, als es der Stiftungszweck vorgibt. Daneben können - bei Verzicht auf die steuerlichen Vorteile der Gemeinnützigkeit - auch für andere Kunden als die Einrichtungen der Stiftung und ihrer gemeinnützigen Tochter Leistungen erbracht werden. Diese Konstruktion aus Stiftung mit diversen Tochtergesellschaften begünstigt einerseits die Wirtschaftlichkeit der Stiftungseinrichtungen und bietet eine hohe Flexibilität. Die Tätigkeit der Tochtergesellschaften kann andererseits der Stiftung beispielsweise durch Gewinnausschüttung zugutekommen. Diese stellen dann Erträge der Vermögensverwaltung dar.

Sollen Geschäftsfelder klar getrennt werden, ist die gemeinnützige Stiftung als Rechtsform somit nicht hinderlich. Während für die Tätigkeit im Sozialbereich die Stiftungsform dem Wohlfahrtswerk kei-

\section{Wohlfahrtswerk für Baden-Württemberg: der Auftrag}

\section{$\mathbb{S} 1$ Aufgaben, Gemeinnützigkeit}

(1) Die Stiftung verfolgt ausschließlich und unmittelbar gemeinnützige und mildtätige Zwecke im Sinne des Abschnitts "Steuerbegünstigte Zwecke« der Abgabenordnung. Sie ist selbstlos tätig und verfolgt nicht in erster Linie eigenwirtschaftliche Zwecke.

(2) Im einzelnen hat die Stiftung folgende Aufgaben:

1. Verwendung der Erträge des Stiftungsvermögens sowie Zuwendungen von dritter Seite für Zwecke der Wohlfahrtspflege;

2. Verwaltung ihr übertragener Stiftungen, Vermächtnisse und Zuwendungen für wohltätige Zwecke nach Maßgabe des Willens der Zuwendenden;

3. Gründung, Unterhaltung und Betrieb von Wohlfahrtseinrichtungen;

4. Erprobung fortschrittlicher Methoden sozialer Arbeit;

5. Gewährung von Unterstützung und Beihilfen in besonderen Notfällen;

6. Herausgabe von Veröffentlichungen auf dem Gebiet der Wohlfahrtspflege;

7. Aus-, Fort- und Weiterbildung sozialer und pflegerischer Fachkräfte.

(3) Die Mittel der Stiftung dürfen nur für die satzungsmäßigen Zwecke verwendet werden. Die Mitglieder der satzungsmäßigen Organe dürfen keine Gewinnanteile erhalten.

(4) Keine Person darf durch Verwaltungsausgaben, die dem Zweck der Stiftung fremd sind oder durch unverhältnismäßig hohe Vergütungen begünstigt werden.

Auszug aus der Stiftungsurkunde »Wohlfahrtswerk für Baden-Württemberg" nen unternehmerischen Nachteil bringt, gibt es Vorteile, die bei anderen gemeinnützigen Unternehmensformen fehlen.

Ein weiterer Satzungszweck des Wohlfahrtswerks für Baden-Württemberg ist die »Verwaltung ihr übertragener Stiftungen, Vermächtnisse und Zuwendungen für wohltätige Zwecke nach Maßgabe des Zuwendenden «. Unterstiftungen sind an einen eingetragenen Verein oder eine gemeinnützige Gesellschaft der beschränkten Haftung nicht möglich. Diese können nur Spenden entgegennehmen. Vorteil der Zustiftungen ist, dass der Geldbetrag üblicherweise größer ist als eine normale Spende. Der Satzungszweck des Wohlfahrtswerks für Baden-Württemberg ist für diese Aufnahme von Unterstiftungen oder die Verwaltung anderer Stiftungen geeignet. Ohne diesen Satzungszweck würde es sich bei der Verwaltung der Unterstiftung um einen wirtschaftlichen Geschäftsbetrieb mit allen Konsequenzen handeln.

Die Altenhilfeeinrichtungen des Wohlfahrtswerks für Baden-Württemberg sehen sich stetig verschärfenden wirtschaftlichen Rahmenbedingungen und einem intensiven Wettbewerb ausgesetzt. Die Unternehmensführung zielt darauf, dass sich die Einrichtungen den Entgelten wirtschaftlich tragen. Dies schmälert nicht die Bedeutung von Spenden und Fundraising-Aktivitäten für die Einrichtungen. Aus Entgelten nicht finanzierte zusätzliche Angebote oder Anschaffungen werden damit möglich, beispielsweise die dementengerechte Gestaltung des Gartens, der »Urlaub vom Pflegeheim» oder die technische Ausrüstung für Gemeinschaftsveranstaltungen. Die Erfahrungen zeigen, dass die Verbundenheit mit einem konkreten Haus oder einem Projekt wichtig ist.

\section{Die Stiftung als Innovator}

Die Stiftungssatzung des Wohlfahrtswerks für Baden-Württemberg enthält einen für das Selbstverständnis der eigenen Einrichtungen wichtigen Zweck: »die Erprobung fortschrittlicher Methoden sozialer Arbeit «. Das Innovationsverständnis kann sich auf Unternehmensstrukturen, Prozessoptimierung, neue Produkte oder Dienstleistungen richten. Das Wohlfahrtswerk konzentrierte sich auf letzteres und das stetige Streben nach innovativen 
Angeboten immer durch die eigenen Einrichtungen umgesetzt. Beispielsweise war die im Jahre 1981 eingerichtete Tagespflege für Senioren die erste Einrichtung dieser Art in Süddeutschland. Und mit dem erstmaligen Angebot von Betreutem Wohnens für Senioren im Jahr 1987 war nach außen. Wirtschaftliche und organisatorische Dinge müssen objektiv abgewogen werden. Die Unternehmenskultur dagegen wird geprägt von Menschen, von einer oder mehreren Leitfiguren und durch von Menschen geborene Leitideen. Welche Ideen es sind, dass diese kultiviert

\section{"Stiftungen können auch andere Rechtsformen nutzen: durch Gründung von Tochtergesellschaften"}

das Wohlfahrtswerk für Baden-Württemberg deutschlandweit Pionier. Seit dem Jahr 2000 wird mit einer Wohngemeinschaft für pflegebedürftige Menschen Erfahrung gesammelt, was zunächst mit finanziellen Verlusten einherging. Die Bedeutung dieser Erfahrungen für die inhaltliche Auseinandersetzung mit dem Angebot in den anderen eigenen Einrichtungen und für die Entwicklung der Altenhilfestrukturen allgemein rechtfertigt in diesem Fall die Verwendung von Stiftungsmitteln. Damit sind die Einrichtungen des Wohlfahrtswerks für BadenWürttemberg bei Innovationen nicht allein auf Fördermittel und Spendenakquise angewiesen. Hauptsächlich werden neue Ideen aber aus dem laufenden Betrieb heraus finanziert und müssen sich nach einer Anlaufzeit selbst tragen.

Zur Verbreitung der Erkenntnisse aus der Praxis des Wohlfahrtswerks für Baden-Württemberg und aus eigenen modellhaften Projekten werden Fachtagungen veranstaltet, die dem Stiftungszweck der "Aus-, Fort- und Weiterbildung sozialer und pflegerischer Fachkräfte« dienen. Die Ergebnisse der Fachtagungen werden in Dokumentationen aufbereitet, die dem letzten hier zu nennenden Stiftungszweck dienen, der »Herausgabe von Veröffentlichungen auf dem Gebiet der Wohlfahrtspflege «. Auch die Herausgabe dieser Zeitschrift zählt dazu.

\section{Leitfiguren und Leitideen prägen die Unternehmenskultur}

Die Umsetzung von Stiftungszielen ist nicht allein von der Rechtsform abhängig, sondern auch von der Unternehmenskultur. Diese wirkt nach innen und und über Jahre hinweg gepflegt werden ist wiederum ein Ergebnis der Unternehmenskultur.

Im Wohlfahrtswerk für Baden-Württemberg zeigen sich die Leitideen durch die Jahrhunderte hinweg konstant, lediglich die Formen der Umsetzung wandeln sich. Die von Königin Katharina formulierte Maxime lautet in heutigen Worten ausgedrückt »Hilfe zur Selbsthilfe«. Dieses Leitbild wurde gepflegt und ist bis heute für das Ethos und die Unternehmensplanung im Wohlfahrtswerk relevant. Beispielsweise wäre die Abkehr von pauschalen Wohnheimangeboten und Entwicklung des Betreuten Wohnens mit dem Motto »So selbständig wie möglich, so viel Hilfe wie nötig « ohne diese Überzeugung nicht denkbar gewesen.

Für die künftige Ausrichtung sind die Ideen der führenden Köpfe ausschlaggebend. Diesbezüglich hat die Frage, wer im Aufsichtsgremium sitzt und wie dessen Besetzung erfolgt, eine sehr praktische Relevanz. Denn Aufgabe der Mitglieder des Aufsichtsgremiums ist die Auswahl des oder der Vorstände, die wiederum Personalverantwortung tragen. Die Personalentscheidung ist noch aus ganz anderen Gründen wesentlich: Die handelnden Personen müssen Vertrauen bewirken, ohne das Dienstleistung im Sozialbereich vielfach gar nicht erbracht werden kann.

\section{Fazit}

Die Umwandlung der fast zwei Jahrhunderte alten Zentralleitung der Wohltätigkeitsvereine in das heutige Wohlfahrtswerk für Baden-Württemberg darf als gelungenes Beispiel einer verantwor- tungsvollen Privatisierung bezeichnet werden. In den über drei Jahrzehnten seit der Stiftungsgründung wurde die betriebliche Tätigkeit konsequent auf Nutzerinteressen und Wirtschaftlichkeit ausgerichtet.

\section{Anmerkungen}

(1) Siehe z. B. Senatsbeschluss der Stadt Hamburg vom 22. August 2006 zum Verkauf der Anstalt öffentlichen Rechts "pflegen \& wohnen « mit zwölf Pflegezentren zum 1. Januar 2007 an die Vitanas Hamburg $\mathrm{GmbH}$ für 65 Millionen Euro (www.vitanas.de/presse/Mitteilungen/2006-08-22_PM_Senat_HH_Verkaufpw) oder der Verkauf von 475 stationären Pflegeplätzen in Pflegheimen und 53 Altenwohnungen der kommunalen Kieler Betreuungs- und Pflegedienste gGmbH an die Unternehmensgruppe Senator Senioren-Residenzen (Lübeck) für 5,5 Millionen Euro im Jahr 2003 (Altenheim 2/2004, S. 10).

(2) Regierungskommission Deutscher Corporate Governance Kodex: Deutscher Corporate Governance Kodex in der Fassung vom 21. Mai 2003.

(3) Diakonisches Werk der Evangelischen Kirche in Deutschland: Diakonischer Corporate Governance Kodex (DGK). Stand Oktober 2005. Beschlossen auf der Diakonischen Konferenz 2005 in Rummelsberg.

(4) Besonders bei eingetragenen Vereinen findet sich noch häufig die Struktur einer Besetzung des Vorstands mit Ehrenamtlichen und eine hauptamtliche Geschäftsführung. Diese ist teilweise Mitglied des Vorstands, teilweise auch unterhalb der Vorstandsebene angesiedelt.

(5) Die Mitgliedschaft im Paritätischen Wohlfahrtsverband bringt im Gegensatz zur Mitgliedschaft beispielsweise in Diakonie oder Caritas keine von Dachverbandsseite vorgegebenen Tarifbedingungen mit sich. 